\title{
Two New Rapidly Rotating ON Stars Found with LAMOST
}

\author{
Guang-Wei Li (李广伟) ${ }^{1}$ (D) and Ian D. Howarth ${ }^{2}$ (D) \\ ${ }^{1}$ Key Laboratory of Space Astronomy and Technology, National Astronomical Observatories, Chinese Academy of Sciences, Beijing 100101, People's Republic of \\ China; lgw@bao.ac.cn \\ ${ }^{2}$ Department of Physics \& Astronomy, University College London, Gower St., London WC1E 6BT, UK \\ Received 2019 October 29; revised 2019 November 22; accepted 2019 November 22; published 2020 January 10
}

\begin{abstract}
The ON stars are a rare subtype of $\mathrm{O}$ stars of uncertain origin. We report two new, rapidly rotating ON stars found in data acquired with the Large Sky Area Multi-Object Fiber Spectroscopic Telescope. LS I +61 28 is an ON8.5 $\mathrm{Vn}$ dwarf with a projected equatorial rotational velocity of $v_{\mathrm{e}} \sin i \simeq 298 \mathrm{~km} \mathrm{~s}^{-1}$, while HDE 236672 is an ON9 IVn subgiant with $v_{\mathrm{e}} \sin i \simeq 253 \mathrm{~km} \mathrm{~s}^{-1}$. The former is the first rapidly rotating ON dwarf to be found, and the latter is only the third ON subgiant. The luminosity classes of non-supergiant ON stars appear to be influenced by the axial inclination angle $i$ : the rapidly rotating giants are close to equator-on, while ON dwarfs with lower $v_{\mathrm{e}}$ sin $i$ values are viewed more nearly pole-on. Combining parallaxes and proper motions from Gaia Data Release 2 with radial-velocity measurements, we investigate the kinematics of non-supergiant ON stars and infer that the dynamics, rapid rotation, and surface nitrogen characteristics may all be consequences of binary interaction.
\end{abstract}

Unified Astronomy Thesaurus concepts: Stellar rotation (1629); O stars (1137)

\section{Introduction}

The systematics of peculiar $\mathrm{C}$ and $\mathrm{N}$ spectral morphologies in $\mathrm{OB}$ stars were first put on a secure footing by Walborn (1971), who introduced the OBC and OBN classification notations. In particular, O-type stars with N III $\lambda \lambda 4634-4640$ 4642 absorption stronger than C III $\lambda \lambda 4647-4650-4652$ are classified as ON (Walborn 1971; Sota et al. 2011). Because the $\mathrm{N}$ III triplet is prone to emission at high temperatures and low gravities, and disappears at low temperatures, this diagnostic is most effective for late-O and early-B stars, which dominate the known OBN sample. ${ }^{3}$ However, the ON phenomenon has been identified in stars with classifications as early as $\mathrm{O} 2$, based on N IV lines (Walborn et al. 2004), and across all luminosity classes (Walborn et al. 2004, 2016).

OBN morphology has been understood to be the result of surface exposure of CNO-burning products since the work of Lester (1973), which is an inference supported by the observation that observed surface $\mathrm{CNO}$ ratios fall between equilibrium values expected for partial $\mathrm{CN}$ (and complete $\mathrm{CNO}$ ) burning (Martins et al. 2015a; Carneiro et al. 2019). The evolutionary processes giving rise to enrichment of surface nitrogen have been discussed since Walborn (1970). As a class, ON stars have systematically higher $v_{\mathrm{e}} \sin i$ values than morphologically normal counterparts (Howarth \& Smith 2001; Martins et al. 2015b), with a subset of notably rapidly rotating ONn stars (Walborn 2003). Rapid rotation may induce mixing, which can transport processed material from the stellar core to the surface (Maeder \& Meynet 2000; Przybilla et al. 2010); homogeneous evolution is possible for the most massive stars (see, e.g., Walborn et al. 2004; Przybilla et al. 2010; Martins et al. 2015a; Carneiro et al. 2019). Binary interaction can both spin up an accretor (the initially less massive component; Packet 1981) and enrich its surface nitrogen (Langer 2012). Sana et al. (2012) found that $71 \%$ of O-type stars are born in binaries that undergo a subsequent mass exchange, lending credence to a binary-interaction channel.

\footnotetext{
3 N III $\lambda \lambda 4511-4515$ was used as an indicator to select ON stars by Bisiacchi et al. (1982), but Walborn et al. (2016) suggested that the equivalent width ratio of $(\lambda \lambda 4511-15) /(\lambda 4650)$ is a more reliable discriminant.
}

All the ON dwarfs ${ }^{4}$ listed by Walborn et al. (2011) are relatively slow rotators, and those authors suggested that enriched surface nitrogen observed in stars that have not had time to expose core-processed material may be the result of mass transfer. By contrast, all the ON giants listed by Walborn et al. (2011) are rapid rotators (ONn stars). Rotation can therefore provide us with clues to an understanding of the nature and origin of ON stars.

In this paper, we report the discovery of two new rapidly rotating ON stars, including a first ONn dwarf. Section 2 summarizes the Large Sky Area Multi-Object Fiber Spectroscopic Telescope (LAMOST) spectra of these stars. In Section 3, by combining parallaxes and proper motions provided by Gaia Data Release 2 (DR2; Gaia Collaboration et al. 2018) with radial-velocity data from Walborn et al. (2011), we investigate the kinematics of the late-type ON stars and draw inferences on their origin.

\section{Data \\ 2.1. LAMOST}

LAMOST (otherwise known as the Guoshoujing Telescope) has 4000 focal-plane fibers feeding 16 separate spectrographs (Wang et al. 1996; Su \& Cui 2004; Cui et al. 2012; Luo et al. 2012; Zhao et al. 2012). It has been conducting low- and medium-resolution spectral surveys since 2011 November and 2018 October, respectively, with resolving powers of $\sim 1800$ (a wavelength range of $\lambda \lambda 3690-9100$ ) and $\sim 7500$ (simultaneous coverage of $\lambda \lambda 4920-5360$ and $6290-6860 \AA$ ). The current data release (LAMOST DR7; http://dr7.lamost.org) contains 10 million low-resolution spectra of $\sim 7$ million objects and 2 million medium-resolution spectra.

The standard LAMOST stellar spectral-template library contains no O stars, so we added standards from Maíz Apellániz et al. (2016) and then used the augmented library to identify the O-type

\footnotetext{
4 For clarity, we note explicitly that we use the terms "dwarf," "giant," etc., as indicators of spectral morphology; we are not concerned with ON supergiants in this paper. In this context, these terms are primarily indicators of apparent surface gravity and not necessarily of evolutionary status; see Section 3.
} 


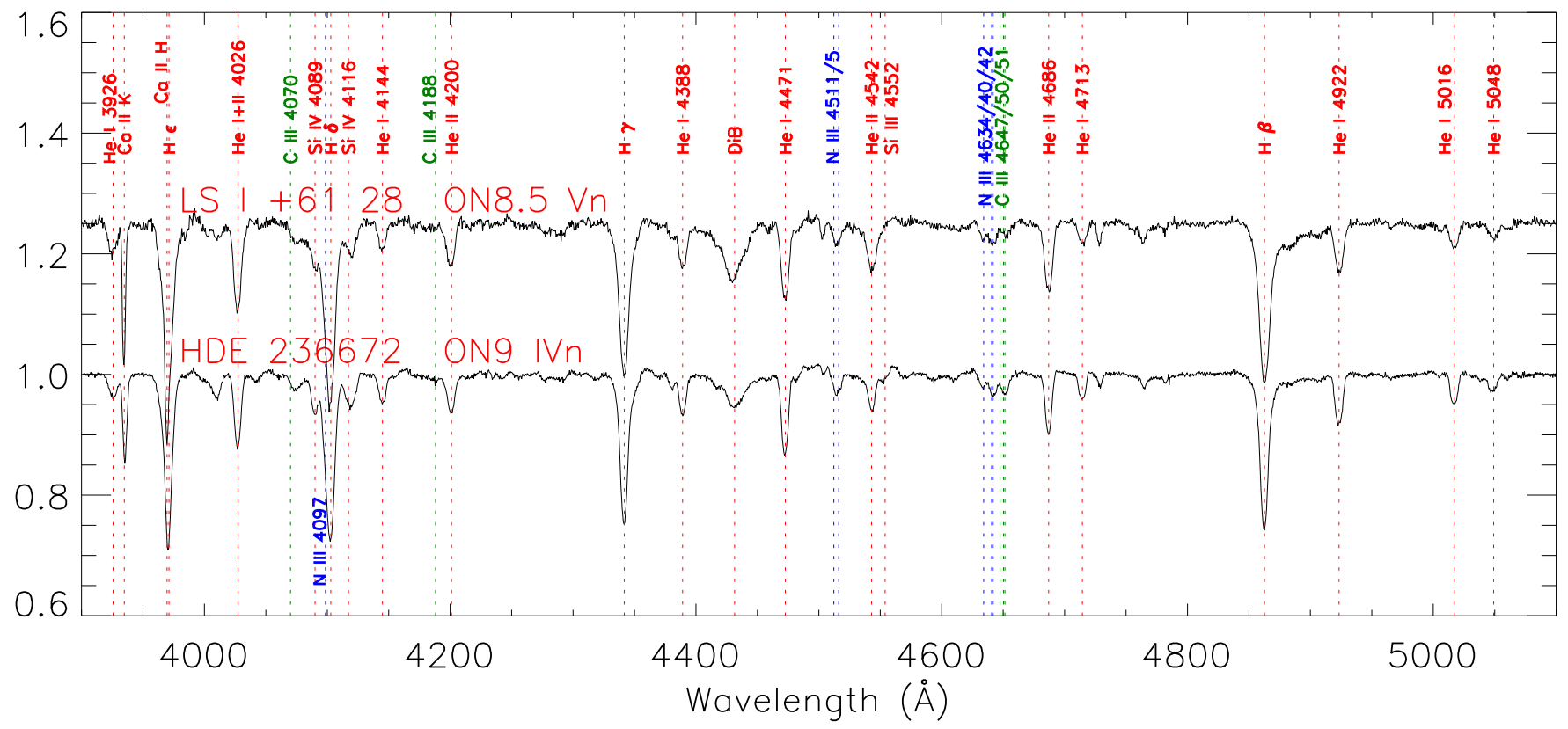

Figure 1. LAMOST low-resolution spectra of LS I +61 28 and HDE 236672. N III and C III lines are indicated by blue and green dotted lines, respectively.

Table 1

LAMOST Spectra for LS I +61 28 and HDE 236672

\begin{tabular}{lccc}
\hline \hline $\begin{array}{l}\text { Target } \\
\text { Name }\end{array}$ & $\begin{array}{c}\text { Date } \\
\text { (YYYY MM DD) }\end{array}$ & SpecID & $\begin{array}{c}\text { Resolving } \\
\text { Power }\end{array}$ \\
\hline \multirow{2}{*}{ LS I +61 28 } & 2015 Oct 28 & NGC7788_305073 & 1800 \\
& 2017 Dec 3 & NGC77880105073 & 7500 \\
& 2017 Oct 30 & HIP1174470105073 & 7500 \\
\hline \multirow{4}{*}{ HDE 236672 } & 2014 Sep 9 & NGC457_303097 & 1800 \\
& 2014 Oct 7 & NGC457_303097 & 1800 \\
& 2014 Dec 7 & NGC457_303097 & 1800 \\
& 2016 Dec 13 & NGC457_303097 & 1800 \\
& 2017 Oct 4 & HIP60270104022 & 7500 \\
& & NGC4570103097 & 7500 \\
\hline
\end{tabular}

stars in the low-resolution spectra. We examined, by eye, all spectra of the resulting O-star candidates and found only two new ONn stars: LS I +61 28 and HDE 236672.

\subsection{The Spectra of $L S I+6128$ and HDE 236672}

One low-resolution and two medium-resolution spectra are available for LS I +6128 in the LAMOST data release, with four low-resolution and two medium-resolution spectra for HDE 236672. The observations were obtained on different nights over the course of several years (see Table 1), but there is no evidence for radial-velocity variations in these data, so there is no indication that either star is a close binary (see below).

Figure 1 shows the low-resolution spectra. The N III $\lambda \lambda 4634-4640-4642$ lines in both stars are stronger than C III $\lambda \lambda 4647-4650-4652$, which indicates that these are ON stars, though C III $\lambda \lambda 4647-4650-4652$ is obviously weaker in LS I +61 28 than HDE 236672; N III $\lambda \lambda 4511-4515$ is strong in both stars. The helium lines are broad and shallow, indicating very rapid rotation.

We classify LS I +61 28 as an O8.5 star from the He II $\lambda 4542 /$ He I $\lambda 4388$ and He II $\lambda 4200 / \mathrm{He} \mathrm{I} \lambda 4144$ line ratios, following the precepts of Sota et al. (2011). The weakness of He I $\lambda 4713$ relative to He II $\lambda 4686$ indicates a dwarf; hence, we classify LS I +61 28 as ON8.5 Vn - the first ON Vn star to be identified.

He II $\lambda 4542$ is slightly weaker than He I $\lambda 4388$ in the spectrum of HDE 236672, while He II $\lambda 4200$ is stronger than He I $\lambda 4144$, giving an $\mathrm{O} 9$ temperature class. He I $\lambda 4713$ is weak, but stronger than that of LS I +6128 , leading to an ON9 IVn classification.

We merged the medium-resolution spectra of each star to improve the signal to noise and determined radial and projected equatorial rotational velocities $\left(v_{\mathrm{R}}\right.$ and $\left.v_{\mathrm{e}} \sin i\right)$ from $\mathrm{He} \mathrm{I}$ $\lambda 5016$. We chose this line as it is relatively strong in both stars, with a symmetrical profile.

To establish $v_{R}$, the line center was determined by fitting a modified Gaussian function and linear local continuum:

$$
f(\lambda)=a_{0} \exp \left\{-\frac{1}{a_{3}}\left|\frac{\lambda-a_{1}}{a_{2}}\right|^{a_{3}}\right\}+a_{4}+a_{5} \lambda
$$

where $a_{0-5}$ are fit coefficients, $\lambda$ is the wavelength, and the $f(\lambda)$ is the flux; $v_{\mathrm{R}}$ follows from $a_{1}$.

We estimated $v_{\mathrm{e}} \sin i$ (again from $\lambda 5016$ ) as follows:

1. We fitted Equation (1) to the He I $\lambda 5016$ profile in the LAMOST medium-resolution spectrum of TYC 1323$1592-1^{5}$ to estimate $P(\lambda)$, which is the rectified intrinsic line profile without rotation.

2. For an assumed $v_{\mathrm{e}} \sin i$, we fitted the function

$$
\begin{aligned}
F\left(\lambda, v_{\mathrm{e}} \sin i\right)= & b_{1} \times\left(P(\lambda) \otimes G\left(\lambda, v_{\mathrm{e}} \sin i\right)\right. \\
& +b_{2}+b_{3} \lambda
\end{aligned}
$$

to $S(\lambda)$, which is the observed He I $\lambda 5016$ profile for each ON star. Here, $\otimes$ is the convolution operator; $G\left(\lambda, v_{\mathrm{e}} \sin i\right)$ is the rotational broadening function (Gray 2005); and $b_{1}, b_{2}$, and $b_{3}$ are fit parameters.

\footnotetext{
5 TYC 1323-1592-1 (=LS 36; Stephenson \& Sanduleak 1971) is an O8 VZ star newly identified here. It has the narrowest line profiles among the LAMOST medium-resolution spectra of $\mathrm{O}$ dwarfs, hence, we assume $v_{\mathrm{e}} \sin i \simeq$ 0 ; the exact value is of little consequence, as long as it is small (which is clearly the case).
} 

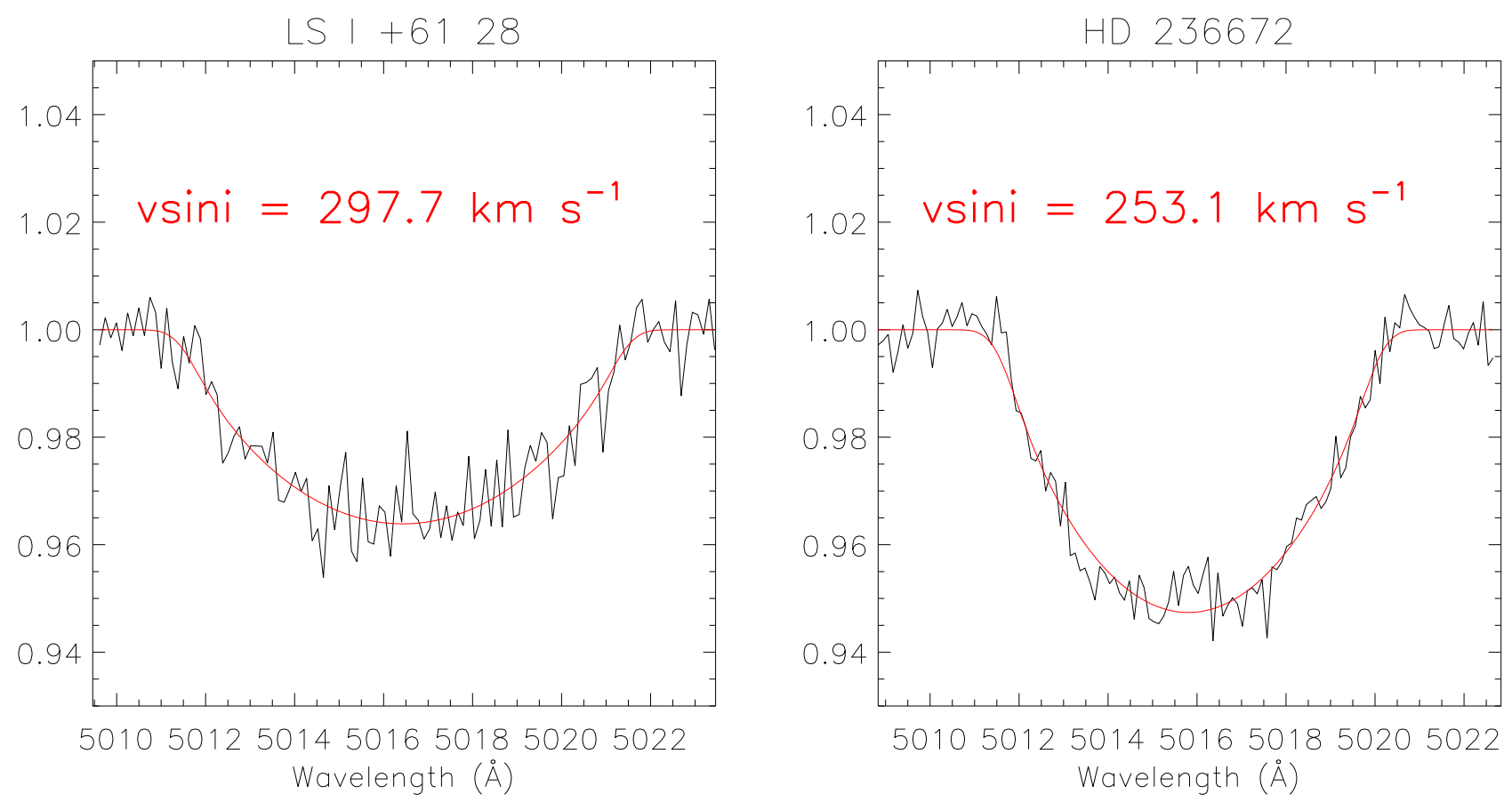

Figure 2. He I $\lambda 5016$ profiles of LS I +6128 and HDE 236672. The red lines are the best-fit rotationally broadened models.

3. A figure of merit, $\xi=\sum_{\lambda}\left(F\left(\lambda, v_{\mathrm{e}} \sin i\right)-S(\lambda)\right)^{2}$, was calculated for trial $v_{\mathrm{e}} \sin i$ values from 180 to $400 \mathrm{~km} \mathrm{~s}^{-1}$ at steps of $7 \mathrm{~km} \mathrm{~s}^{-1}$. A refined best-fit $v_{\mathrm{e}} \sin i$ was finally obtained by fitting a third-order polynomial to the $11 \xi$ values about the smallest one for each star.

To estimate (internal) uncertainties on the velocities, we assumed the best-fit model to be representative of the true profile, added Gaussian noise matching the observed spectra, and measured $v_{\mathrm{R}}$ and $v_{\mathrm{e}} \sin i$ as above, repeating the process $10^{4}$ times. Our final results for $v_{\mathrm{R}}$ and $v_{\mathrm{e}} \sin i$ are: $-36.8 \pm 7.4$, $297.7 \pm 7.8 \mathrm{~km} \mathrm{~s}^{-1}$ (LS I +6128$)$ and $-74.5 \pm 2.6,253.1 \pm$ $3.1 \mathrm{~km} \mathrm{~s}^{-1}$ (HDE 236672).

The He I $\lambda 5016$ rectified observations and adopted models are shown in Figure 2. If macroturbulence were more important in the target stars than in TYC 1323-1592-1, our $v_{\mathrm{e}} \sin i$ values may be upper limits (Howarth et al. 1997; Simón-Díaz \& Herrero 2007), although rotational broadening clearly dominates for the rapid rotators.

We also measured the radial velocity of $\mathrm{He}$ I $\lambda 5016$, along with other lines of hydrogen and helium, in the low-resolution spectra at our disposal, and find no evidence for radial-velocity variability in excess of $\sim 20 \mathrm{~km} \mathrm{~s}^{-1}$ (i.e., $\sim 0.1$ of the resolution element).

\section{Kinematics of Galactic ON Stars}

Gaia DR2 provides parallaxes and proper motions with unprecedented precision for more than a billion stars (Gaia Collaboration et al. 2018). By combining this information with radial velocities, we can determine the kinematics of ON stars in the Galaxy. In addition to the two new ONn stars reported here, we investigate all those $\mathrm{ON}$ stars satisfying the following criteria: (i) not classified as a supergiant, (ii) parallax accuracy of $\varpi / \sigma_{\varpi}>5$, and (iii) a radial velocity reported in Table 2 of Walborn et al. (2011). This sample is summarized in Table 2. Other than for their rotation velocities, the dozen sample stars have similar spectral characteristics and similar effective temperatures (and thus may have arisen through similar processes).

We used Markov Chain Monte Carlo methods to infer the distance and velocity for each star (following Luri et al. 2018), using uninformative priors. The emcee algorithm (ForemanMackey et al. 2013) was first employed to obtain $10^{4}$ samples of distances and proper motions (in R.A., $\alpha$, and decl., $\delta$ ). Second, we generated $10^{4}$ radial-velocity instances from the Gaussian distribution of $v_{\mathrm{R}} \pm \sigma_{v_{\mathrm{R}}}$. Walborn et al. (2011) did not tabulate radial-velocity uncertainties; we assumed $\sigma_{v_{\mathrm{R}}}=$ $10 \mathrm{~km} \mathrm{~s}^{-1}$ (adopting our own estimates for LS I +61 28 and HDE 236672). For stars for which Walborn et al. (2011) gave two measurements, we took the average $v_{\mathrm{R}}$ value. The astropy package was used to convert from the International Celestial Reference System to the Galactocentric system for each sample, finally giving probability distributions for the position and peculiar velocity in the Galactocentric system for each star (adopting values for the solar Galactocentric distance and peculiar velocity, and the Galactic velocity curve, from Eilers et al. 2019).

Figure 3 shows the resulting distributions of peculiar Galactocentric radial, circular, and vertical velocities $\left(V_{\mathrm{r}}, V_{\mathrm{c}}\right.$, and $\left.V_{\mathrm{z}}\right)$ and the height from the Galactic plane ( $z$ ) for the two new ON stars. To characterize these asymmetric distributions, we fit the peaks with a quartic polynomial, taking the maximum of the function as the point estimator, $r_{\text {est }}$ (with 68\% errors shown in Figure 3 and tabulated in Table 2). Results for the wider ON sample are summarized in Figure 4 and are included in Table 2. They show that:

1. Each of the stars in Table 2 has a rotational velocity greater than the median of $\sim 90 \mathrm{~km} \mathrm{~s}^{-1}$ found for all $\mathrm{O}$ stars (and for $\mathrm{O}$ dwarfs alone) by Howarth et al. (1997).

2. The ON stars with giant classifications all have $v_{\mathrm{e}} \sin i>200 \mathrm{~km} \mathrm{~s}^{-1}$, while slower rotators occur only 

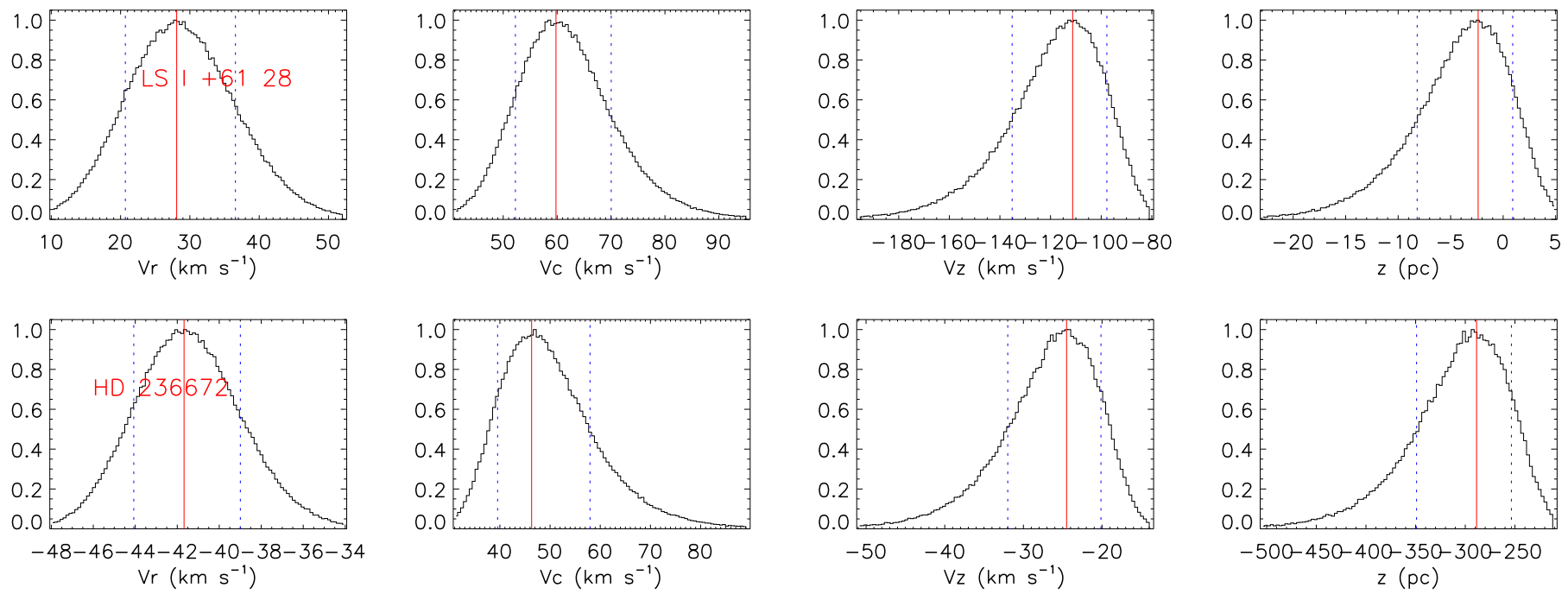

Figure 3. Distributions of peculiar kinematics (left to right: $V_{r}, V_{c}, V_{z}$, and $z$ ) in the Galactocentric system for LS I +6128 (top row) and HDE 236672 (bottom row). In each panel, the red line is the position of point estimator $r_{\text {est }}$, while blue dotted lines show $68 \%$ bounds.

Table 2

Kinematic Parameters for the ON-star Sample

\begin{tabular}{|c|c|c|c|c|c|c|c|c|c|}
\hline Name & $\begin{array}{c}\text { No. } \\
\text { (Figure 4) }\end{array}$ & SpT & $\begin{array}{c}v_{\mathrm{e}} \sin i \\
\left(\mathrm{~km} \mathrm{~s}^{-1}\right)\end{array}$ & $\begin{array}{c}z \\
(\mathrm{pc})\end{array}$ & $\begin{array}{c}V_{\mathrm{r}} \\
\left(\mathrm{km} \mathrm{s}^{-1}\right)\end{array}$ & $\begin{array}{c}V_{\mathrm{c}} \\
\left(\mathrm{km} \mathrm{s}^{-1}\right)\end{array}$ & $\begin{array}{c}V_{\mathrm{z}} \\
\left(\mathrm{km} \mathrm{s}^{-1}\right)\end{array}$ & $\begin{array}{c}V \\
\left(\mathrm{~km} \mathrm{~s}^{-1}\right)\end{array}$ & $\begin{array}{l}\text { Vel. } \\
\text { var? }\end{array}$ \\
\hline HD 13268 & 1 & ON8.5 IIIn & 310 & $-116.2_{-13.6}^{+9.9}$ & $-72.1_{-8.0}^{+8.0}$ & $-38.5_{-6.2}^{+6.2}$ & $0.4_{-2.2}^{+1.8}$ & $81.7_{-9.9}^{+10.0}$ & $\mathrm{C}$ \\
\hline HD 89137 & 2 & ON9.7 II-III(n) & 202 & $323.6_{-33.9}^{+65.4}$ & $59.0_{-7.9}^{+12.8}$ & $83.6_{-13.4}^{+22.6}$ & $14.3_{-1.7}^{+2.2}$ & $102.7_{-13.1}^{+24.5}$ & $\mathrm{~V}$ ? \\
\hline HD 91651 & 3 & ON9.5 IIIn & 310 & $-32.8_{-6.5}^{+4.4}$ & $-6.2_{-0.8}^{+0.9}$ & $76.1_{-9.9}^{+10.0}$ & $-8.3_{-2.2}^{+1.6}$ & $77.0_{-9.8}^{+9.8}$ & SB2 \\
\hline HD 102415 & 4 & ON9 IIInn & 376 & $41.0_{-0.8}^{+1.0}$ & $-5.5_{-1.8}^{+1.7}$ & $33.3_{-9.9}^{+9.9}$ & $-4.7_{-1.1}^{+0.9}$ & $33.5_{-8.8}^{+9.9}$ & $\mathrm{~V}$ ? \\
\hline HD 117490 & 5 & ON9.5 IIInn & 375 & $84.0_{-4.4}^{+6.7}$ & $-73.1_{-8.3}^{+7.0}$ & $35.2_{-9.5}^{+9.3}$ & $7.0_{-0.7}^{+0.6}$ & $81.5_{-4.0}^{+6.1}$ & SB2? \\
\hline HDE 236672 & 8 & ON9 IVn & 253 & $-288.6_{-60.4}^{+35.0}$ & $-41.7_{-2.4}^{+2.7}$ & $46.3_{-6.8}^{+11.6}$ & $-24.5_{-7.5}^{+4.4}$ & $66.5_{-4.7}^{+10.3}$ & $\mathrm{C}$ ? \\
\hline HD 201345 & 9 & ON9.5 IV & 95 & $-507.5_{-147.9}^{+71.9}$ & $1.2_{-2.3}^{+3.6}$ & $52.1_{-9.7}^{+10.0}$ & $-97.1_{-28.6}^{+13.5}$ & $110.2_{-12.9}^{+27.1}$ & $\mathrm{SB} 2$ \\
\hline LS I +61 28 & 10 & ON8.5 Vn & 298 & $-2.4_{-5.8}^{+3.3}$ & $28.1_{-7.4}^{+8.5}$ & $59.8_{-7.5}^{+10.2}$ & $-111.3_{-23.9}^{+13.5}$ & $128.6_{-15.1}^{+26.3}$ & $\mathrm{C}$ ? \\
\hline HD 12323 & 11 & ON9.5 V & 130 & $-253.4_{-47.3}^{+28.1}$ & $1.8_{-8.7}^{+9.3}$ & $10.6_{-5.9}^{+5.9}$ & $-69.2_{-13.8}^{+8.2}$ & $70.9_{-8.1}^{+13.9}$ & SB \\
\hline HD 48279 & 12 & ON8.5 V & 137 & $-6.4_{-7.9}^{+4.1}$ & $0.4_{-9.4}^{+9.3}$ & $56.5_{-5.4}^{+6.6}$ & $-4.0_{-3.5}^{+1.9}$ & $55.8_{-4.1}^{+7.7}$ & $\mathrm{C}$ \\
\hline
\end{tabular}

Note. Spectral types (column 3) and binary indicators (column 10) adopted from Walborn et al. (2011), except HDE 236672 and LS I +61 28 (this paper). Projected rotational velocities are from Martins et al. (2015b), Howarth et al. (1997), and this paper. In column 9, $V=\sqrt{V_{\mathrm{r}}^{2}+V_{\mathrm{c}}^{2}+V_{\mathrm{z}}^{2}}$.

among the dwarfs and subgiants (all of which have $v_{\mathrm{e}} \sin i<300 \mathrm{~km} \mathrm{~s}^{-1}$ ).

3. All of the dwarfs/subgiants have peculiar space velocities of $V>50 \mathrm{~km} \mathrm{~s}^{-1}$, and hence are runaways by conventional criteria (e.g., Blaauw 1961); the same is true of four of the seven giants (with the remaining three having $V \gtrsim 30 \mathrm{~km} \mathrm{~s}^{-1}$ ).

4. While the runaway ON giants have large $V_{r}$ and/or $V_{c}$ velocities, their $V_{z}$ velocities are all small, and appear unexceptional - that is, the favored direction of motion of the runaway ON giants is within $\sim 10^{\circ}$ of the Galactic plane.

5. In contrast to the ON giants, three of the five dwarf/ subgiant stars show $V_{z}$ values much greater than $\sqrt{V_{r}^{2}+V_{c}^{2}}$.

Item (1) is in accord with the now well-established fact that ON stars, as a class, rotate faster than morphologically normal counterparts (Howarth \& Smith 2001; Martins et al. 2015b), while item (2) suggests a causal link between $v_{\mathrm{e}} \sin i$ and spectral morphology; since rapid rotation reduces the equatorial surface gravity, the spectra of stars viewed more nearly equator-on show more giant-like qualities.

The remaining characteristics are suggestive of a degree of correlation between $v_{\mathrm{e}} \sin i$ and kinematics. If runaway velocities were acquired through close gravitational interactions in parental clusters, it is unclear why such a relationship should occur, other than by chance. We therefore consider instead the circumstances of a binary system disrupted by the eruption of the primary as a supernova.

We suppose that the orbital and rotational angular-momentum vectors in such a binary are initially aligned, at an angle of $i_{\text {gal }}$ to the plane of the Galaxy, and that the space motion of the ejected star remains close to the initial orbital plane. Then $0 \leqslant V_{z} \leqslant V \cos \left(i_{\mathrm{gal}}\right)$ and $i_{\mathrm{gal}}<i<90^{\circ}$ (depending on the orbital phase at disruption); hence for large $i_{\text {gal }}$, both small $V_{z}$ 

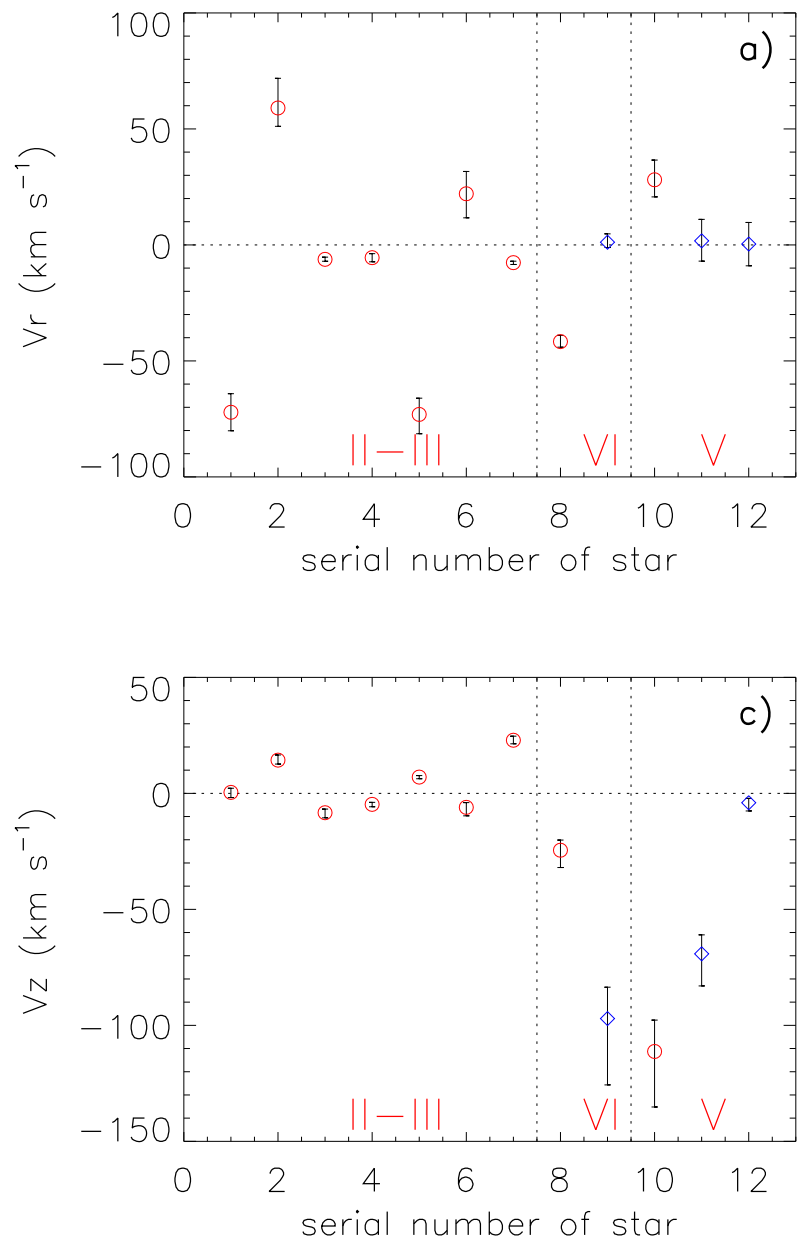
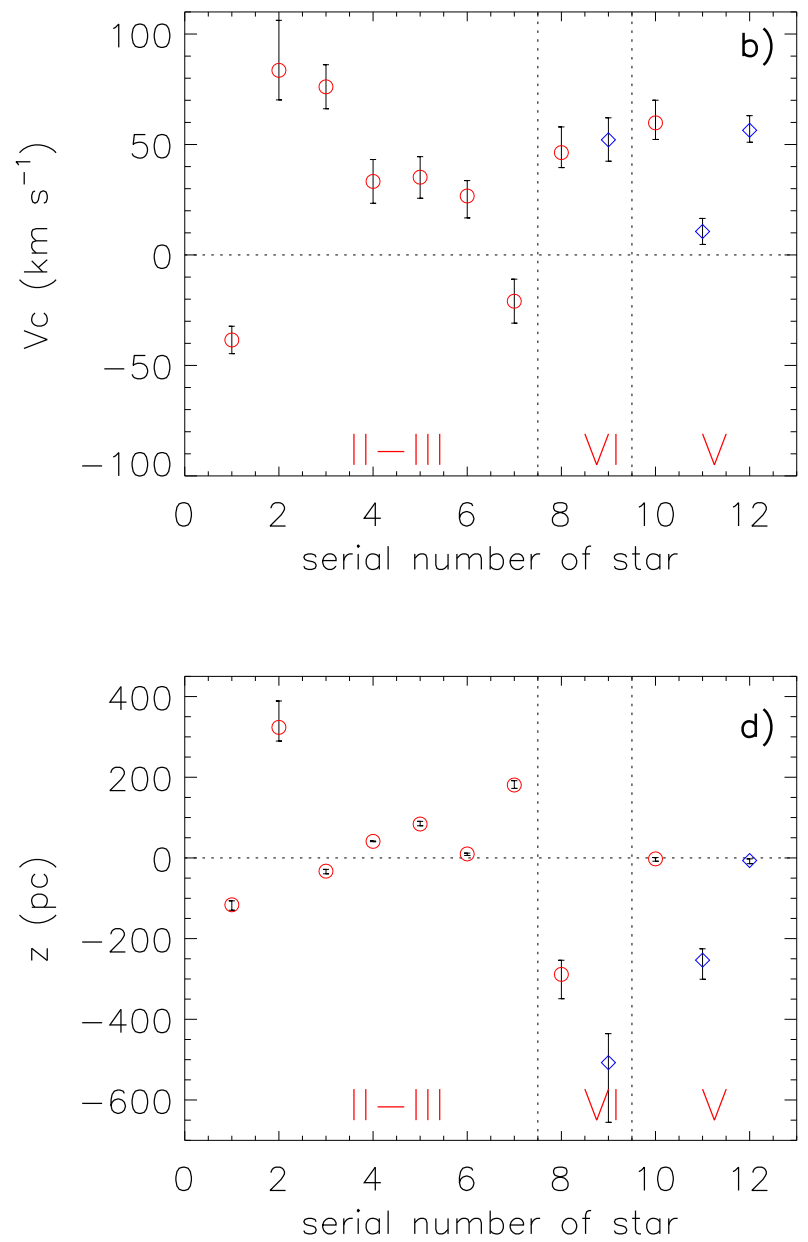

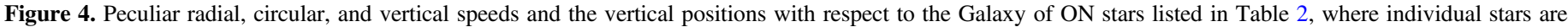

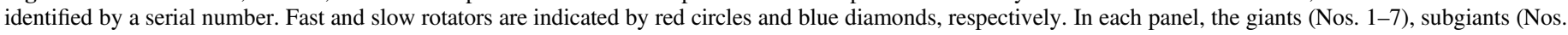
8 and 9), and dwarfs (Nos. 10-12) are separated by vertical dotted lines.

and large $i$ ensue (maximizing $v_{\mathrm{e}} \sin i$ ), corresponding to the $\mathrm{ON}$ giants. At smaller $i_{\text {gal }}$ the potential for $V_{z}$ and $i$ being decoupled increases (becoming independent at $i_{\text {gal }}=0^{\circ}$ ), accommodating the $\mathrm{ON}$-dwarf characteristics (noting that the mean value of $i_{\text {gal }}$ is as large as $60^{\circ}$ for randomly orientated initial orbits, so the tendency is still toward relatively high projected rotational velocities).

We therefore speculate that the near-main-sequence ON stars are products of mass transfer in binary systems and that at least some of them were ejected by the explosion of an initially more massive, faster-evolving primary. The progenitors of the $\mathrm{ON}$ stars are then the mass gainers, and could be sped up, potentially to near-critical rotation, by accreting only a few per cent of their original mass from the primaries (Packet 1981), accounting for the generally rapid rotation.

Accretion could also be responsible for the surface enhancement of nitrogen, through photospheric contamination by transferred products. Conservative mass-transfer models suggest that the surface-nitrogen abundance of the mass gainer may be increased by factors of three to six (Wellstein et al. 2001; Langer 2012), although Song et al. (2018) found that accretion does not necessarily result in nitrogen enrichment on the surface of the mass gainer. However, rapid rotation can still induce mixing, which is thought to increase with rotation and with the stellar mass (Maeder \& Meynet 2000). For a massive star that undergoes chemically homogeneous evolution, the mixing induced by rotation can transport the products of nucleosynthesis to the surface and fresh hydrogen fuel into the core. As a result, the star can have a longer main-sequence lifetime than would otherwise be the case, with enhanced surface nitrogen (Vázquez et al. 2007; Brott et al. 2011). Langer et al. (2008) proposed that the abundance of surface nitrogen can be enriched up to 1 dex for a star that is spun up by accretion.

Unsolved puzzles remain. First, although the kinematics $-v_{\mathrm{e}} \sin i$ correlation suggests that disruption of binary systems may be a significant factor in the lifetime of many near-main-sequence ON stars, a substantial fraction of the objects listed in Table 2 are reported to show radial-velocity variability at some level (see Walborn et al. 2011, and references therein). In most cases, the supporting evidence is rather weak and, according to the current $S_{\mathrm{B}}^{9}$ compilation (Pourbaix et al. 2004), extends to a confirmed orbit in only one case: HD 12323 (Stickland \& Lloyd 2001). The mass function for this SB1 system is as small as $0.01 M_{\odot}$, suggesting that the (current) secondary has $m_{2} \sin \left(i_{\text {orb }}\right) \simeq 1-2 M_{\odot}$. The origins of such objects remain enigmatic.

Second, the timescales $\tau_{z}=v / V_{z}$ are large for several stars, exemplified by HD 89137, for which $\tau_{z} \simeq 80 \mathrm{Myr}$ - an order of magnitude greater than typical main-sequence lifetimes for single late-O stars. The problem of the origin of high-latitude early-type stars is not, however, confined to ON stars (see, e.g., the O9.5 IIInn star HD 93521; Howarth \& Reid 1993). 


\section{Conclusion}

We report the following results:

1. We found two new ONn stars from LAMOST lowresolution spectra, LS I +61 28 and HDE 236672. LS I +61 28 is the first rapidly rotating ON dwarf to be reported, while HDE 236672 is the third ONn subgiant.

2. From medium-resolution LAMOST spectra, we obtained radial and rotational velocities for both stars: $-36.8 \pm 7.4$ and $297.7 \pm 7.8 \mathrm{~km} \mathrm{~s}^{-1}$ for LS I +6128 and $-74.5 \pm 2.6$ and $253.1 \pm 3.1 \mathrm{~km} \mathrm{~s}^{-1}$ for HDE 236672 .

3. By combining radial velocities with parallaxes and proper motions from Gaia DR2, we propose that the ON stars originate from binary interactions, and the surfacenitrogen enrichments probably result from rotationally induced mixing.

4. The morphological luminosity class of an ON star is determined by the axial inclination angle $i$ : giants with high $v_{\mathrm{e}} \sin i$ values are viewed edge-on, while ON dwarfs with smaller projected equatorial velocities are more nearly pole-on.

This research is supported by the National Natural Science Foundation of China (NSFC; grant No. 11673036). The Guoshoujing Telescope (LAMOST) is a National Major Scientific Project built by the Chinese Academy of Sciences. Funding for the project has been provided by the National Development and Reform Commission. LAMOST is operated and managed by the National Astronomical Observatories, Chinese Academy of Sciences.

Facilities: Guoshoujing Telescope (LAMOST), Gaia.

Software: astropy (Astropy Collaboration et al. 2013), emcee (Foreman-Mackey et al. 2013).

\section{ORCID iDs}

Guang-Wei Li (李广伟) (1) https://orcid.org/0000-00017515-6307

Ian D. Howarth (1) https://orcid.org/0000-0003-3476-8985

\section{References}

Astropy Collaboration, Robitaille, T. P., Tollerud, E. J., et al. 2013, A\&A, 558, A33

Bisiacchi, G. F., Lopez, J. A., \& Firmani, C. 1982, A\&A, 107, 252
Blaauw, A. 1961, BAN, 15, 265

Brott, I., de Mink, S. E., Cantiello, M., et al. 2011, A\&A, 530, A115

Carneiro, L. P., Puls, J., Hoffmann, T. L., Holgado, G., \& Simón-Díaz, S. 2019, A\&A, 623, A3

Cui, X.-Q., Zhao, Y.-H., Chu, Y.-Q., et al. 2012, RAA, 12, 1197

Eilers, A.-C., Hogg, D. W., Rix, H.-W., \& Ness, M. K. 2019, ApJ, 871, 120

Foreman-Mackey, D., Hogg, D. W., Lang, D., \& Goodman, J. 2013, PASP, 125,306

Gaia Collaboration, Brown, A. G. A., Vallenari, A., et al. 2018, A\&A, 616, A1

Gray, D. F. 2005, The Observation and Analysis of Stellar Photospheres (3rd ed.; Cambridge: Cambridge Univ. Press)

Howarth, I. D., \& Reid, A. H. N. 1993, A\&A, 279, 148

Howarth, I. D., Siebert, K. W., Hussain, G. A. J., \& Prinja, R. K. 1997, MNRAS, 284, 265

Howarth, I. D., \& Smith, K. C. 2001, MNRAS, 327, 353

Langer, N. 2012, ARA\&A, 50, 107

Langer, N., Cantiello, M., Yoon, S.-C., et al. 2008, in IAU Symp. 250, Massive Stars as Cosmic Engines, ed. F. Bresolin, P. Crowther, \& J. Puls (Cambridge: Cambridge Univ. Press), 167

Lester, J. B. 1973, ApJ, 185, 253

Luo, A.-L., Zhang, H.-T., Zhao, Y.-H., et al. 2012, RAA, 12, 1243

Luri, X., Brown, A. G. A., Sarro, L. M., et al. 2018, A\&A, 616, A9

Maeder, A., \& Meynet, G. 2000, ARA\&A, 38, 143

Maíz Apellániz, J., Sota, A., Arias, J. I., et al. 2016, ApJS, 224, 4

Martins, F., Hervé, A., Bouret, J.-C., et al. 2015a, A\&A, 575, A34

Martins, F., Simón-Díaz, S., Palacios, A., et al. 2015b, A\&A, 578, A109

Packet, W. 1981, A\&A, 102, 17

Pourbaix, D., Tokovinin, A. A., Batten, A. H., et al. 2004, A\&A, 424, 727

Przybilla, N., Firnstein, M., Nieva, M. F., Meynet, G., \& Maeder, A. 2010, A\&A, 517, A38

Sana, H., de Mink, S. E., de Koter, A., et al. 2012, Sci, 337, 444

Simón-Díaz, S., \& Herrero, A. 2007, A\&A, 468, 1063

Song, H., Wang, J., Song, F., et al. 2018, ApJ, 859, 43

Sota, A., Maíz Apellániz, J., Walborn, N. R., et al. 2011, ApJS, 193, 24

Stephenson, C. B., \& Sanduleak, N. 1971, Publication of the Warner and Swasey Observatory (Cleveland, OH: Case Western Reserve Univ.)

Stickland, D. J., \& Lloyd, C. 2001, Obs, 121, 1

Su, D.-Q., \& Cui, X.-Q. 2004, ChJAA, 4, 1

Vázquez, G. A., Leitherer, C., Schaerer, D., Meynet, G., \& Maeder, A. 2007, ApJ, 663, 995

Walborn, N. R. 1970, ApJL, 161, L149

Walborn, N. R. 1971, ApJL, 164, L67

Walborn, N. R. 2003, in ASP Conf. Ser. 304, CNO in the Universe, ed. C. Charbonnel, D. Schaerer, \& G. Meynet (San Francisco, CA: ASP), 29

Walborn, N. R., Maíz Apellániz, J., Sota, A., et al. 2011, AJ, 142, 150

Walborn, N. R., Morrell, N. I., Barbá, R. H., \& Sota, A. 2016, AJ, 151, 91

Walborn, N. R., Morrell, N. I., Howarth, I. D., et al. 2004, ApJ, 608, 1028

Wang, S.-G., Su, D.-Q., Chu, Y.-Q., Cui, X., \& Wang, Y.-N. 1996, ApOpt, 35,5155

Wellstein, S., Langer, N., \& Braun, H. 2001, A\&A, 369, 939

Zhao, G., Zhao, Y.-H., Chu, Y.-Q., Jing, Y.-P., \& Deng, L.-C. 2012, RAA, 12, 723 